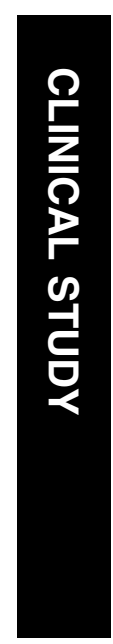

\section{The effect of etanercept on Graves' ophthalmopathy: a pilot study}

${ }^{1}$ Department of Oculoplastic and Orbital Surgery, Rotterdam Eye Hospital, Rotterdam, The Netherlands

${ }^{2}$ Department of Internal Medicine, Rotterdam Eye Hospital, Rotterdam, The Netherlands

${ }^{3}$ Department of Nuclear Medicine, Erasmus Medical Centre, Rotterdam, The Netherlands

${ }^{4}$ Department of Internal Medicine, Erasmus Medical Centre, Rotterdam, The Netherlands

Correspondence:

D Paridaens, Department of Oculoplastic and Orbital Surgery, The Rotterdam Eye Hospital, Schiedamsevest 180, 3011 BH Rotterdam, The Netherlands.

Tel: + 31104017777 ;

Fax: + 31104017655

E-mail: a.paridaens@ worldonline.nl

Received: 25 May 2004 Accepted in revised form: 4 October 2004

Published online:

19 November 2004

\begin{abstract}
Purpose To assess the effect of etanercept, an antitumour necrosis factor (anti-TNF) drug, on clinical signs in Graves' ophthalmopathy (GO). Methods A total of 10 consecutive patients
\end{abstract} (seven female, three male) with recent-onset, active, mildly-to-moderately severe GO were treated with subcutaneous injections of $25 \mathrm{mg}$ etanercept (Enbrel ${ }^{\mathbb{R}}$ ) twice weekly during $\mathbf{1 2}$ weeks. The average age was 50 years (range: 39-59 years). The average duration of GO was 4 months (range: 2-6 months). All patients were at least 2 months euthyroid with medication. The mean pretreatment clinical activity score (CAS) was 4 (range: 3-6). Results The mean CAS prior to treatment was 4 , at 6 weeks 2.6, and at 12 weeks 1.6. The mean ophthalmopathy index (OI) prior to treatment was 5.8, at 6 weeks 4.8 , and at 12 weeks 4.4. Thus, after 12 weeks of treatment the mean CAS had decreased by $2.4 \pm 1.3$ $(60 \%)$, and the mean OI had decreased by $1.4 \pm 1.2(24 \%)$. The main decrease of the CAS and OI was noted at 6 weeks of treatment. The difference was particularly evident on softtissue changes, including periocular chemosis and redness. The mean exophthalmometry values did not change. In all, $60 \%$ percent of patients reported moderate to marked improvement. In three patients, the GO flared up again after cessation of treatment. There were no serious adverse events or side effects during a mean follow-up time of 18 months.

Conclusions This study strongly suggests that etanercept may suppress the clinical signs in GO, but randomized controlled trials are needed to further evaluate the effect of antiTNF treatment and to compare its side-effects with those of corticosteroids. Eye (2005) 19, 1286-1289. doi:10.1038/sj.eye.6701768; published online 19 November 2004
D Paridaens ${ }^{1}$, WA van den Bosch'1, TL van der Loos $^{2}$, EP Krenning ${ }^{3}$ and PM van Hagen ${ }^{4}$
Keywords: TNF; etanercept; ophthalmopathy; thyroid

\section{Introduction}

Although the pathogenesis of Graves' ophthalmopathy (GO) remains incompletely understood, cytokines appear to play a major role in the initiation and maintenance of this disorder. ${ }^{1}$ One of these cytokines, tumour necrosis factor- $\alpha$ (TNF- $\alpha$ ), has been shown to be of importance in $\mathrm{GO}^{2,3}$ and in other autoimmune diseases, including rheumatoid arthritis (RA). ${ }^{4}$

Clinical trials have shown a beneficial effect of anti-TNF treatment in $\mathrm{RA}^{5,6}$ and, recently, in acute uveitis. ${ }^{7}$ However, another study on patients with uveitis treated with methotrexate showed that relapse of the disease could not be prevented by anti-TNF treatment. ${ }^{8}$ To date, there has been no published report on the effect of such treatment in GO. This prompted us to perform a pilot trial in a small series of patients.

We used etanercept $\left(\right.$ Enbrel $\left.^{\circledR}\right)$, which is a dimeric fusion protein consisting of the extracellular ligand-binding portion of the human $75 \mathrm{kDa}$ (p75) tumour necrosis factor receptor (TNFR) linked to the Fc portion of human IgG1. It is produced by recombinantDNA technology and consists of 934 amino acids and has a molecular weight of approximately $150 \mathrm{kDa}$. Etanercept binds specifically to TNF and blocks its interaction with cell surface TNF receptors. ${ }^{5,6}$ The clinical use of etanercept is contraindicated in patients with sepsis and a known hypersensitivity to this drug or any of its components. Treatment with etanercept may result in the formation of autoimmune antibodies. It has been shown to cause intestinal obstruction in patients suffering from Crohn's disease. ${ }^{5}$ Anti-TNF treatment may affect host defences against infections and 
malignancies since TNF mediates inflammation and modulates cellular immune responses.

\section{Methods}

We treated 10 patients with mild or moderately severe GO with subcutaneous injections of $25 \mathrm{mg}$ etanercept $\left(\right.$ Enbrel $\left.^{\mathbb{R}}\right)$ twice weekly during 12 weeks. We included patients who were at least 2 months euthyroid with medication (suppression-substitution therapy), who had developed their eye signs during the last six months and who showed active ophthalmopathy, that is, who scored at least three points in the clinical activity score (CAS). ${ }^{9}$ In this score, each of the following 10 symptoms and signs is assigned one point: orbital pain at rest, pain during eye movements, redness of the eyelid(s), redness of the conjunctiva, eyelid swelling, conjunctival oedema, swelling of the caruncle, $>2 \mathrm{~mm}$ proptosis, $>1$ line decrease of visual acuity, $>5$ degrees decrease of ocular motility.

We excluded patients with a known allergy to etanercept, with infections, with sepsis, with other autoimmune disorders, with a (history of) malignancy, patients below 18 years of age, pregnant patients, and patients who had been treated with immunosuppressive drugs or retrobulbar irradiation. Patients were excluded if during the study period thyroid surgery or a therapeutic dosage of radioactive iodine was indicated, or if during the study period hypothyroidism or hyperthyroidism (plasma T3, T4, and TSH values outside the normal reference values) developed. We also excluded patients who showed signs of advanced optic nerve compression, such as a decreased (pinhole) vision, a relative afferent papillary defect, decreased colour vision, abnormal visual field findings, or visual-evoked potentials (VEP).

The study was approved by the institutional review board, and patients gave informed consent for their participation.

Ophthalmic examination included visual acuity measurement, assessment of periocular changes, slitlamp biomicroscopy, intraocular pressure measurement, and fundus examination. Exophthalmometry was performed with a Hertel exophthalmometer (Rodenstock). In addition, visual fields, and VEP were assessed. Orbital computerized tomography (CT) scans were made. Orthoptic examination included assessment of diplopia and ductions.

Efficacy of treatment was determined using the following scoring systems:

1. Clinical activity score (CAS). ${ }^{9}$

2. Ophthalmomopathy index (OI) ${ }^{10}$ based on the NOSPECS classification. Each category of class $2-6$ is scored as follows: grade $a=1, b=2, c=3$. The sum $=$ OI.

3. Exophthalmometry values.

4. Patient self-assessment. Patients were asked to evaluate subjectively whether their situation after treatment showed deterioration (marked, moderate, minimal), no change, or improvement (minimal, moderate, marked).

Full ophthalmic and orthoptic examination, including assessment of the CAS, OI, and exophthalmometry values, was performed at intake, after 6 weeks, and after 12 weeks of treatment.

\section{Results}

We included seven female and three male patients with an average age of 50 years (range: $39-59$ years). The mean CAS, OI, and exophthalmometry values at intake, at 6 weeks and at 12 weeks of treatment are summarized in Table 1. After treatment, the CAS had decreased by, on average $60 \%$, and the OI by $24 \%$. The difference was particularly evident on soft-tissue changes, including periocular chemosis and redness (Figure 1). All patients reported improvement, especially during the first 6 weeks of treatment: two patients had marked improvement, four had moderate improvement, and four had minimal improvement. At intake, six patients suffered from diplopia. In two of them, the degree of diplopia diminished from continuous to discontinuous diplopia. In one patient, the discontinuous diplopia resolved after treatment. In the other three, there was no improvement of continous diplopia. None of the four patients without diplopia at intake developed diplopia during treatment. After treatment, an improvement of elevation up to 10 degrees was noted in two patients, deterioration in one (4 degrees), and no change in the other seven. Overall, there was no change of the other ductions (depression, adduction, and abduction).

In all 10 patients, the visual acuity did not change during treatment. Two patients showed early signs of optic nerve compression at intake (normal visual acuity but a slightly abnormal VEP and slight visual field changes). In both patients, the VEP as well as the visual fields proved normal after 3 months. We found no average change in exophthalmometry after treatment.

No (serious) adverse events related to the administration of etanercept occurred during or following treatment, with a mean follow-up of 18 months. ${ }^{11}$ None of the 10 patients were discontinued from the study. One patient experienced mild, transient injection site reaction (erythema). After cessation of treatment, periocular inflammatory signs recurred within 2-6 weeks in three patients. One of these was euthyroid 
Table 1 Clinical findings before and after etanercept treatment in 10 patients with mild-to-moderate, active Graves' ophthalmopathy

\begin{tabular}{lccc}
\hline $\begin{array}{l}\text { Time-point in study } \\
\text { (in weeks) }\end{array}$ & $\begin{array}{c}\text { Clinical activity score (CAS), } \\
\text { mean (range) }\end{array}$ & $\begin{array}{c}\text { Ophthalmopathy index (OI), } \\
\text { mean (range) }\end{array}$ & $\begin{array}{c}\text { Exophthalmometry } \\
\text { (in mm), mean (range) }\end{array}$ \\
\hline$T=0$ & $4(3-6)$ & $5.8(3-10)$ & $21.9(15-30)$ \\
$T=6$ & $2.6(0-6)$ & $4.8(4-7)$ & $21.8(15-30)$ \\
$T=12$ & $1.6(0-4)$ & $4.4(3-7)$ & $21.9(15-28)$ \\
Difference $(T=12)-(T=0)$ & -2.4 & -1.4 & 0 \\
(\% difference) & $(60 \%)$ & $(24 \%)$ & $(0 \%)$ \\
$(\mathrm{SD})$ & $( \pm 1.3)$ & $( \pm 1.2)$ & $( \pm 1.6)$ \\
\hline
\end{tabular}
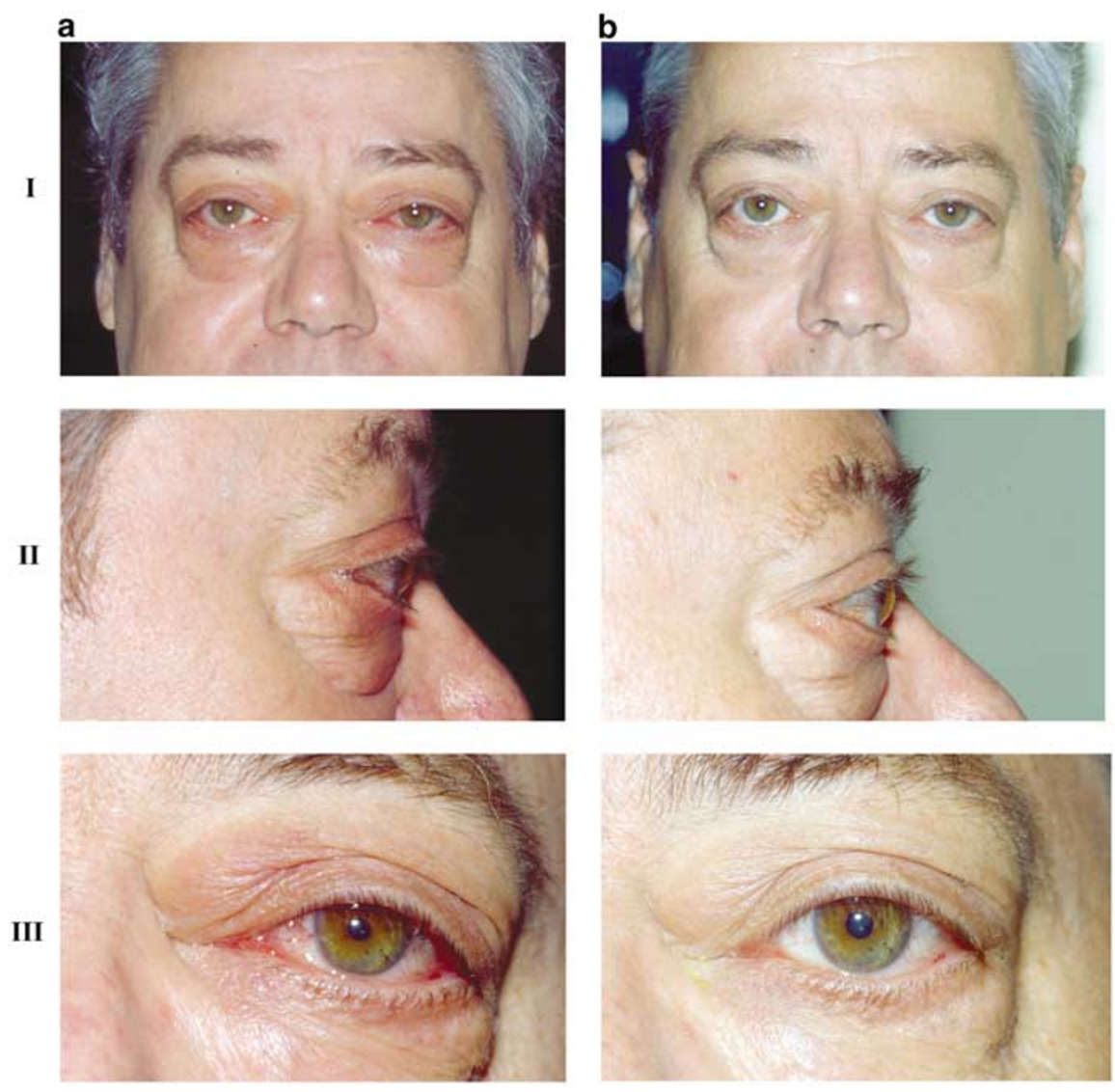

Figure 1 (a) Pretreatment appearance of a 54-year-old male patient with recent-onset, active Graves' ophthalmopathy. I: frontal view; II: lateral view right eye; III: frontal view left eye. (b) Appearance after 12 weeks of treatment with etanercept. Note the reduction of periocular swelling and redness. I: frontal view; II: lateral view right eye; III: frontal view left eye.

at the end of treatment but became hypothyroid shortly after.

\section{Discussion}

This is, to our knowledge, the first report on the clinical use of a TNF- $\alpha$ antagonist in GO. In the majority of patients with active, recent-onset disease, who were treated with etanercept during 12 weeks, we noted a clinical improvement, with an emphasis on the soft- tissue signs. This improvement occurred mainly in the first 6 weeks and remained stable until the end of treatment. Since this was an uncontrolled study, we cannot rule out that the improvement was due to the natural course of this disease. This, however, seems unlikely for two reasons: Firstly, $60 \%$ of patients treated with etanercept showed moderate to marked clinical improvement. In a small, controlled study on the effect of antioxidant agents in $\mathrm{GO}^{12}$ it was found that only about $30 \%$ of patients using placebo treatment clinically 
improved in the study period of 3 months. Secondly, the disease activity increased after cessation of treatment in three out of 10 cases, albeit that one of these three became hypothyroid.

While treatment with corticosteroids may be even more effective in reducing the inflammatory symptoms in patients with GO, prolonged use often causes sideeffects, such as diabetes, osteoporosis, and Cushing's syndrome. Therefore, there is a need for alternative medication that causes fewer side effects. Patients in this study showed a good tolerance to etanercept without noticeable adverse reactions. However, with a follow-up of 18 months, long-term side effects may still develop. It is important to realize that the long-term effects of etanercept treatment on serious infections, autoimmune diseases, and malignancies are unknown.

In conclusion, this pilot study on a small series of patients with GO strongly suggests that etanercept suppresses the clinical signs in patients with active ophthalmopathy without noticeable short-term side effects. A randomized prospective study is needed to see if etanercept (or other TNF-antagonists) prove sufficiently effective in reducing the inflammatory symptoms of GO, if they can be administered safely for a prolonged period, and if these side effects compare favourably to those of corticosteroids.

\section{Acknowledgements}

This work was approved by the Institutional Review Board of the Rotterdam Eye Hospital.

The authors have no financial interest in this study. This work was supported by the SWOO Research Foundation, Rotterdam and Wyeth Pharmaceuticals, Hoofddorp, The Netherlands. Preliminary results have been presented at the Vancouver Orbital Symposium, Vancouver, Canada, 2002; at the 196th Annual Meeting of the Dutch Ophthalmic Society, Groningen, The

Netherlands, 2002; and at the 20th Annual Meeting of the European Society of Ophthalmic Plastic AND Reconstructive Surgery, Muenster, Germany, 2002.

\section{References}

1 Bartalena L, Marcocci C, Pinchera A. Cytokine antagonists: new ideas for the management of Graves'ophthalmopathy. J Clin Endocrinol Metab 1996; 81: 446-448.

2 Bahn RS. Understanding the immunology of Graves' ophthalmopathy. Is it an autoimmune disease? Endocrinol Metab Clin North Am 2000; 29: 287-296.

3 Krassas GE, Pontikides N, Doukidis D, Heufelder G, Heufelder AE. Serum levels of tumor necrosis factor-alpha, soluble intercellular adhesion molecule-1, soluble vascular cell adhesion molecule-1, and soluble interleukin-1 receptor antagonist in patients with thyroid eye disease undergoing treatment with somatostatin analogues. Thyroid 2001; 11: 1115-1122.

4 Houssiau FA. Cytokines in rheumatoid arthritis. Clin Rheumatol 1995; 14(Suppl 2): 10-13.

5 Moreland LW, Baumgartner SW, Schiff MH, Tindall EA, Fleischmann RM, Weaver AL et al. Treatment of rheumatoid arthritis with a recombinant human tumor necrosis factor receptor (p75)-Fc fusion protein. N Engl J Med 1997; 337: 141-147.

6 Weinblatt ME, Kremer JM, Bankhurst AD, Bulpitt KJ, Fleischmann RM, Fox RI et al. A trial of etanercept, a recombinant tumor necrosis factor receptor: FC fusion protein, in patients with rheumatoid arthritis receiving methotrexate. N Engl J Med 1999; 340: 253-260.

7 El-Shabrawi Y, Hermann J. Anti-tumor necrosis factor-alpha therapy with infliximab as an alternative to corticosteroids in the treatment of human leucocyte antigen B27-associated acute anterior uveitis. Ophthalmology 2002; 109: 2342-2346.

8 Foster CS, Tufail F, Waheed NK, Chu D, Miserocchi E, Baltatzis $\mathrm{S}$ et al. Efficacy of etanercept in preventing relapse of uveitis controlled by methotrexate. Arch Ophthalmol 2003; 121: 437-440.

9 Mourits MP, Koornneef L, Wiersinga WM, Prummel MF, Berghout A, van der Gaag R. Clinical criteria for the assessment of disease activity in Graves' ophthalmopathy: a novel approach. Br J Ophthalmol 1989; 73(8): 639-644.

10 Marcocci C, Bartalena L, Bogazzi F, Bruno-Bossio G, Lepri A, Pinchera A. Orbital radiotherapy combined with high dose systemic glucocorticoids for Graves' ophthalmopathy is more effective than radiotherapy alone: results of a prospective randomized study. J Endocrinol Invest 1991; 14(10): 853-860.

11 Koopman WJ, Moreland LW. Rheumatoid arthritis: anticytokine therapies on the horizon. Ann Intern Med 1998; 128: 231-233.

12 Bouzas EA, Karadimas P, Mastorakos G, Koutras DA. Antioxidant agents in the treatment of Graves' ophthalmopathy. Am J Ophthalmol 2000; 129: 618-622. 